\title{
riccafd
}

Revista Iberoamericana de Ciencias de la Actividad Física y el Deporte

\section{PROGRAMA DE ACONDICIONAMIENTO FÍSICO EN MÚSICOS}

FITNESS PROGRAM IN MUSICIANS

\author{
Gómez Patiño, BS. '; Guerrero Luna, YP ${ }^{1}$; Díaz Fernández, YA ${ }^{1}$ y \\ Vernaza- Pinzón, $\mathbf{P}^{2}$.
}

\footnotetext{
${ }^{1}$ Programa de Fisioterapia, Universidad del Cauca, Colombia. Email: brayangomez10@live.com ${ }_{1}^{1}$ Programa de Fisioterapia, Universidd del Cauca, Colombia. Email: yessicag@unicauca.edu.co ${ }^{1}$ Programa de Fisioterapia, Universidad del Cauca, Colombia: Email: diazf@unicauca.edu.co ${ }^{2}$ Fisioterapeuta, Docente titular programa de Fisioterapia, Universidad del Cauca, Colombia. Email: pvernaza@unicauca.edu.co
}

Código UNESCO: 320403 Salud profesional

Clasificación Consejo de Europa: 14 Fisioterapia y rehabilitación

Recibido el 2 de junio de 2016

Aceptado el 28 de junio de 2016

Correspondencia:

Vernaza- Pinzón, $P$

pvernaza@unicauca.edu.co

Agradecimientos a la Universidad del Cauca por el apoyo en los procesos de Investigación.

\section{RESUMEN}

Objetivo: Determinar el efecto de un programa de acondicionamiento físico en la postura de músicos instrumentistas de la Universidad del Cauca, aplicado durante el año 2015.

Método: Se realizó un estudio cuasi experimental longitudinal descriptivo, en 9 integrantes del Ensamble de Vientos de Metal del programa de Música de la Universidad del Cauca; la información se colecto mediante una ficha en la que se reportaron las características sociodemográficas, los estilos de vida y la fuerza muscular; con el programa Kinovea, se evaluó la postura del cuadrante superior; y luego se aplicó un programa de 
acondicionamiento físico; luego de la aplicación del programa, se revaluó la postura del cuadrante superior y la fuerza muscular.

Resultados: Con la aplicación del programa de acondicionamiento físico, el $100 \%$ de los participantes presentaron reducción en los ángulos de desviación del cuadrante superior, principalmente del ángulo cráneo vertebral y en la alineación de la columna cervico-dorsal en la vista lateral.

Conclusión: Con la aplicación del programa de acondicionamiento físico, se evidencia un cambio clínico positivo manifestado en la disminución de los grados de alteración en los ángulos posturales del cuadrante superior.

PALABRAS CLAVE: Música, Acondicionamiento físico humano, postura, fuerza muscular (Fuente: DeCS)

\section{ABSTRACT}

Objective: To determine the effect of a fitness program in the posture of instrumentalists from the University of Cauca, applied during 2015. Method: A descriptive longitudinal quasi-experimental study was conducted in 9 members of the Wind Ensemble of Metal Music program; The information was collected through a tab that were reported sociodemographic characteristics, lifestyle and muscle strength; with Kinovea program, the position of the upper quadrant was evaluated; and then a fitness program was applied; after the implementation of the program, the position of the upper quadrant and muscle strength was revalued.

Results: With the application of fitness program, $100 \%$ of participants showed a reduction in deviation angles upper quadrant, mainly skull and vertebral angle in the alignment of the cervical-thoracic spine in the side view.

Conclusion: With the application of fitness program, a positive clinical change manifested in decreasing degrees of alteration in postural angles of the upper quadrant evidence.

KEY WORDS: Músic, physical Conditioning, Human, posture, Muscle Strength $(\mathrm{MesH})$ 


\section{INTRODUCCIÓN}

La transformación de la postura, es uno de los sucesos más sorprendentes en la evolución del ser humano, por tanto uno de los más complejos de entender (1). Se comprende como postura, la posición relativa que adoptan las diferentes partes el cuerpo en el espacio, siendo una postura correcta aquella que permite un estado de equilibrio musculo esquelético y de protección a las estructuras corporales (2); una postura incorrecta es el producto de los fallos en la relación entre diversas partes del cuerpo, produciendo dolor e incluso limitación en la actividad y restricción en la participación de manera severa. Estas deficiencias posturales, son objeto de numerosos estudios que buscan entender, prevenir, tratar y mejorar estas derivaciones (3).

La música, es el arte relacionado con la combinación de sonidos vocales o instrumentales como una expresión de la belleza de la forma o de una emoción. Un ensamble de vientos, hace referencia a la conjunción de distintos instrumentos de viento, para lograr un producto colectivo armonioso (4). Y en la medida que se integre postura con la interpretación musical se logrará un óptimo estado de salud en los sistemas corporales, esencialmente el sistema osteomuscular por ser el principalmente afectado. El hecho de usar el cuerpo para la interpretación del instrumento musical, implica una fuerza contráctil adicional de un grupo muscular especifico para el mantenimiento de la postura, la carga de peso y la motricidad fina para la activación sonora del instrumento. Dentro de este sistema corporal, es la postura entonces un elemento fundamental para establecer una armonía cuerpo- instrumento, además de brindar la estabilidad, coordinación y soporte, mientras se ejecutan movimientos de segmentos distales durante la interpretación, puesto que la postura está determinada por: factores biomecánicos y la técnica de interpretación musical (5). Debido al ejercicio de su profesión, los músicos pueden desarrollar padecimientos musculo-esqueléticos (6), cuyos factores de 
riesgo se clasifican en intrínsecos (edad y estilos de vida) y extrínsecos relacionados con su práctica. Todos estos factores de riesgo pueden desencadenar enfermedades ocupacionales por esfuerzo repetitivo y enfermedades del miembro superior relacionada con el trabajo.

Estudios del Instituto de Fisiologia i Medicina de l'Art de Terrassa, reportaron que de un total de 1.612 patologías diagnosticadas a 891 músicos de orquestas, se encontró deficiencia en estructura dorsal en un 5,8\%, y cérvico-braquial con el 4,3\% (7) y entre los factores de riesgo para presentar deficiencias posturales en los músicos de instrumentos de viento, se encuentran: 1. Los prolongados e intensos periodos de practica (8); 2. La técnica, que hace referencia a una secuencia apropiada de movimiento con exactitud total en el roce, el contacto, el soporte, la presión, el golpe, el soplido y 3. La postura durante el tiempo de practica-ensayo, la interpretación de las piezas y la relación cuerpo-instrumento, arte y ergonomía (9).

Con estos antecedentes, se hace importante determinar el efecto de un programa de acondicionamiento físico en la postura de músicos instrumentistas de la Universidad del Cauca, el programa busca educar en el conocimiento de prevención de lesiones osteomusculares, hacer conciencia del cuidado postural para mejorar sus métodos de interpretación y su calidad de vida. Esta intervención, permitirá ampliar el campo de acción de la fisioterapia con un impacto positivo en poblaciones de músicos.

\section{MÉTODOS}

Se realizó un estudio cuasi experimental longitudinal, con músicos pertenecientes al ensamble de vientos de metal de la Universidad del Cauca. Inicialmente la investigación contó con la participación de 11 estudiantes y un docente del Programa de Música, dos sujetos fueron excluidos por inasistencia al $40 \%$ de las actividades programadas; Finalmente fueron incluidos nueve 
sujetos (7 trombonistas, 1 interprete de tuba y 1 de Eufonio). Se entregó a la población estudio, el consentimiento informado, se continuó con la evaluación inicial, en la que se consignaron los datos sociodemográficos (género, edad, estado civil, nivel educativo, años de interpretación musical, instrumento interpretado, talla, peso, índice de masa corporal), aspectos de estilos de vida (consumo de medicamentos, hábitos nocivos, práctica de ejercicio físico, horas de sueño, antecedentes familiares y personales, conocimientos de ergonomía), Medidas antropométricas fuerza muscular funcional de los músculos romboideos y extensores del raquis dorsal, estos grupos musculares fueron escogidos dada la carga y tensión impartida a nivel de los músculos de la cintura escapular durante el proceso de interpretación musical. Luego se realizó la evaluación postural a partir del registro fotográfico individual en los planos lateral, anterior y posterior. El análisis de cada plano se realizó con el programa de edición de videos KINOVEA 0.8.15 software libre, herramienta objetiva para evaluar la alineación articular del cuadrante superior. Este programa principalmente utilizado en medicina del deporte, sin embargo es de gran utilidad en otros ámbitos como son el campo laboral y el ergonómico(10).

Después de estas valoraciones, se procedió a aplicar el programa de intervención terapéutico; el que se desarrollo durante 27 sesiones con una frecuencia de un día por semana y una intensidad de 2 horas. Cada sesión de trabajo constó de una fase de acercamiento, una fase inicial de calentamiento general y específico, una fase central en la cual se realizaron actividades de fuerza y resistencia para músculos extensores de columna dorsal y músculos romboideos; se realizaron actividades para facilitar el control postural, la flexibilidad, la coordinación, el equilibrio, y ejercicios de resistencia aeróbica, con actividades terapéuticas que integraban dos o más de estas cualidades, al final de cada sesión, se realizaron estiramientos y técnicas de relajación.

Una vez finalizado el programa se realizó la evaluación final, reportando los datos de medidas antropométricas y fuerza muscular. Adicionalmente, se 20 PROGRAMA DE ACONDICIONAMIENTO FÍSICO EN MÚSICOS 
realizó un nuevo registro fotográfico utilizando el programa generador de video KINOVEA 0.8.15.

Las consideraciones éticas responden a la resolución 8430 del Ministerio de Salud de Colombia, se solicito al consejo de Facultad de la Facultad de Artes, el aval ético para las evaluaciones y aplicación del programa de intervención terapéutico, el cual fue aceptado de forma escrita el 18 de junio de 2015. Los registros fotográficos fueron entregados a cada uno de los participantes, con el fin de preservar la confidencialidad de la información.

Para la recolección y análisis estadístico de la información, se construyó una base de datos en el programa estadístico IBM SPSS.22. Las variables cualitativas se expresaron en frecuencias y proporciones y las variables cuantitativas se expresaron en medidas de tendencia central y medidas de dispersión. El análisis de la alineación articular se realizó en el software de video de postura KINOVEA, el cual arrojó durante las evaluaciones inicial y final los grados de congruencia articular.

\section{RESULTADOS}

Los tres instrumentos que conforman el Ensamble de Vientos de Metal de la Universidad del Cauca, poseen características similares en cuanto a su interpretación; sin embargo, su actitud postural es particular para cada uno de ellos. En la tuba (ver imagen 1), la columna debe estar en posición completamente vertical, las manos y brazos deben permanecer tan relajados como sea posible, la mano que acciona los pistones siempre debe estar colocada sobre los pistones, en todo momento, la punta de los dedos tiene que ubicarse encima de los pistones para lograr mayor fluidez, la mano que no trabaja con los pistones se utilizará para sostener el instrumento y accionar las bombas cuando sea requerido. 
El trombón (ver imagen 2), necesita columna erguida, fijación de cintura escapulo humeral, fuerza de los brazos para su ejecución, la tarea de sujeción de este con la mano izquierda como derecha implica esfuerzo postura sostenido, en el brazo izquierdo se generan cargas estáticas derivadas del esfuerzo muscular que hay que realizar para soportar el peso del trombón, manteniendo el equilibrio durante más de 15 segundos, la mano se debe colocar formando una " $U$ " abierta con los dedos pulgar e índice y el codo en flexión de $150^{\circ}$; el brazo derecho debe estar en postura forzada dinámica, en flexión de $90^{\circ}$ en las posiciones altas, el codo en flexo extensiones repetitivas. Al ser los ángulos grandes y la frecuencia alta, el riesgo de sufrir daño músculo esquelético también es alto.

En el eufonio (Ver imagen 3), la cabeza se debe mantener alineada para permitir el libre paso del aire a través de la garganta (abierta), de igual manera el tronco y la columna deben estar derechos pero sin tensiones que puedan obstaculizar el correcto funcionamiento del sistema respiratorio. El brazo izquierdo da soporte al instrumento en rotación interna, el brazo derecho es el que manipula los pistones, la columna debe estar correctamente alineada. 


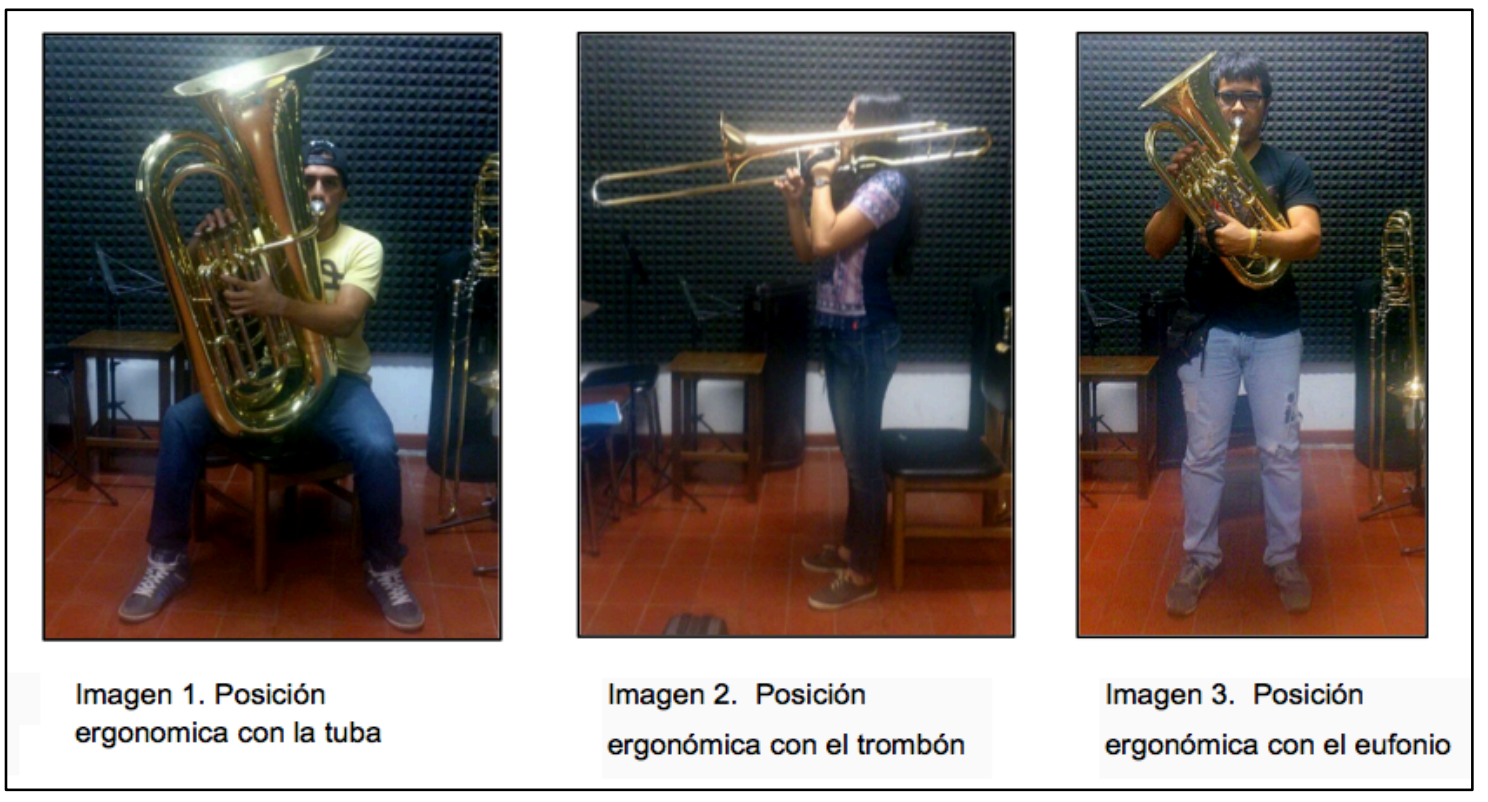

Fuente: Autores

En la tabla 1 se muestran las características sociodemográficas de la población.

Tabla 1. Caracterización sociodemográfica de los integrantes del Ensamble de vientos de metal de la Universidad del Cauca.

\begin{tabular}{llcccccc}
\hline Sujeto & Género & Edad (años) & Estado civil & Peso $(\mathbf{k g})$ & Talla $(\mathbf{m})$ & Régimen de salud & Lugar procedencia \\
\hline Trombón 1 & Masculino & 35 & Soltero & 77 & 1,79 & Contributivo & Risaralda \\
Trombón 2 & Masculino & 23 & Soltero & 79 & 1,77 & Subsidiado & Nariño \\
Trombón 3 & Masculino & 21 & Soltero & 70 & 1,73 & Especial & Nariño \\
Trombón 4 & Masculino & 22 & Soltero & 65 & 1,68 & Subsidiado & Nariño \\
Trombón 5 & Femenino & 22 & Soltera & 64 & 1,68 & Contributivo & Nariño \\
Trombón 6 & Masculino & 23 & Soltero & 85 & 1,80 & Contributivo & Valle \\
Trombón 7 & Masculino & 20 & Soltero & 67 & 1,70 & Especial & Nariño \\
Tuba & Masculino & 20 & Soltero & 69 & 1,75 & Subsidiado & Nariño \\
Eufonio & Masculino & 21 & Soltero & 62 & 1,62 & Subsidiado & Nariño \\
\hline
\end{tabular}

Respecto al estilo de vida y antecedentes de los participantes, el $100 \%$ de los sujetos estudiados, no consumen medicamentos ni bebidas alcohólicas; 5/9 participantes consumen con frecuencia alimentos ricos en grasas y carbohidratos; 6/9 participantes realizan actividad física regular, el promedio 
de horas de sueño es de 8 horas, siendo la mínima 6 y la máxima 10 horas de sueño; respecto a los antecedentes de dolor muscular 3/9 lo presentan en zona de romboides, luego de la intervención solo 1 sujeto reportó dolor en la misma zona. finalmente los conocimientos evaluados sobre prevención de lesiones y ergonomía, arrojo que solo 3 personas tenían conocimientos sobre estos aspectos (Datos no mostrados)

En cuanto a la variable de fuerza muscular para los músculos extensores de columna dorsal, ocho participantes obtuvieron una calificación de $5 / 5$ y uno calificación de 3/5. Para los músculos romboideos, ocho obtuvieron una calificación de 5/5 y 1 calificación de 4/5. Dicha valoración se realizó con la escala de Daniels (3).

En las nueve personas evaluadas, al inicio de la intervención se observaron diferentes alteraciones en el cuadrante superior, en cada una de las vistas analizadas.

Tabla 2. Perfil postural del cuadrante superior de los integrantes del ensamble de vientos de metal antes y después de la intervención. Vista lateral.

\begin{tabular}{|c|c|c|c|c|c|c|c|c|c|c|}
\hline \multirow[t]{2}{*}{ Sujeto } & \multicolumn{2}{|c|}{$\begin{array}{l}\text { Cabeza inclinada } \\
\text { izquierda }\end{array}$} & \multicolumn{2}{|c|}{$\begin{array}{c}\text { Cabeza } \\
\text { inclinada } \\
\text { derecha }\end{array}$} & \multicolumn{2}{|c|}{$\begin{array}{c}\text { Escápula } \\
\text { izquierda alto }\end{array}$} & \multicolumn{2}{|c|}{$\begin{array}{l}\text { Escápula derecha } \\
\text { alta }\end{array}$} & \multicolumn{2}{|c|}{$\begin{array}{c}\text { Tronco } \\
\text { inclinación } \\
\text { izquierda }\end{array}$} \\
\hline & Antes & Después & Antes & Después & Antes & Después & Antes & Después & Antes & Después \\
\hline Trombón 1 & $4^{\circ}$ & $3^{\circ}$ & - & - & $3^{\circ}$ & $1^{\circ}$ & - & - & $1^{\circ}$ & $0^{\circ}$ \\
\hline Trombón 2 & $4^{\circ}$ & $2^{\circ}$ & - & - & $6^{\circ}$ & $2^{\circ}$ & - & - & $2^{\circ}$ & $0^{\circ}$ \\
\hline Trombón 3 & $3^{\circ}$ & $0^{\circ}$ & - & - & $3^{\circ}$ & $5^{\circ}$ & & & $1^{\circ}$ & $0^{\circ}$ \\
\hline Trombón 4 & $1^{\circ}$ & $1^{\circ}$ & - & - & - & - & $0^{\circ}$ & $1^{\circ}$ & $1^{\circ}$ & $0^{\circ}$ \\
\hline Trombón 5 & $1^{\circ}$ & $0^{\circ}$ & - & - & $4^{\circ}$ & $1^{\circ}$ & - & - & $3^{\circ}$ & $1^{\circ}$ \\
\hline Trombón 6 & $2^{\circ}$ & $0^{\circ}$ & - & - & $5^{\circ}$ & $2^{\circ}$ & - & - & $2^{\circ}$ & $0^{\circ}$ \\
\hline Trombón 7 & $2^{\circ}$ & $1^{\circ}$ & - & - & $4^{\circ}$ & $2^{\circ}$ & - & - & $0^{\circ}$ & $0^{\circ}$ \\
\hline Tuba & - & - & $2^{\circ}$ & $1^{\circ}$ & $1^{\circ}$ & $4^{\circ}$ & - & - & $2^{\circ}$ & $1^{\circ}$ \\
\hline Eufónio & 1 & $3^{\circ}$ & - & - & $1^{\circ}$ & $2^{\circ}$ & - & - & $0^{\circ}$ & $0^{\circ}$ \\
\hline
\end{tabular}


En las tablas 2 y 3 , se muestran los resultados arrojados por el software KINOVEA en cada una de las vistas evaluadas, mencionando que cero grados hace referencia a ningún grado de alteración o desviación

En la vista anterior de proximal a distal (tabla 3), se observó luego de la intervención en términos generales reducción del ángulo de desviación de la cabeza y en términos particulares mejores respuesta post-intervención de los trombonistas 5 y 6 con reducción de los ángulos de desviación en la cabeza orientada hacia la izquierda y alineación de los hombros. En la vista posterior (tabla 3), se encontró en general reducción del ángulo de desviación del tronco, y mejoras post-intervención en todos los ítems evaluados del trombonista 2, contrario a esto el trombonista 3 tuvo aumento del ángulo de desviación sobre la escápula izquierda. En la vista lateral derecha (tabla 2) se evidenció mejora post-intervención en el ángulo cráneo vertebral.

Tabla 3. Perfil postural del cuadrante superior de los integrantes del ensamble de vientos de metal antes y después de la intervención. Vista anterior y posterior (Ant*-antes, Des*-Después)

\begin{tabular}{|c|c|c|c|c|c|c|c|c|c|c|c|c|c|c|c|c|}
\hline & \multicolumn{10}{|c|}{ Vista anterior } & \multicolumn{6}{|c|}{ Vista posterior } \\
\hline & \multicolumn{2}{|c|}{$\begin{array}{c}\text { Cabeza } \\
\text { inclinada } \\
\text { izquierda }\end{array}$} & \multicolumn{2}{|c|}{$\begin{array}{c}\text { Cabeza } \\
\text { inclinada } \\
\text { derecha }\end{array}$} & \multicolumn{2}{|c|}{$\begin{array}{l}\text { Escápula } \\
\text { izquierda } \\
\text { alto }\end{array}$} & \multicolumn{2}{|c|}{$\begin{array}{c}\text { Escápula } \\
\text { derecha alta }\end{array}$} & \multicolumn{2}{|c|}{$\begin{array}{c}\text { Tronco } \\
\text { inclinación } \\
\text { izquierda }\end{array}$} & \multicolumn{2}{|c|}{$\begin{array}{c}\text { Cabeza } \\
\text { inclinada } \\
\text { izquierda }\end{array}$} & \multicolumn{2}{|c|}{$\begin{array}{l}\text { Hombro } \\
\text { izquierdo } \\
\text { alto }\end{array}$} & \multicolumn{2}{|c|}{$\begin{array}{c}\text { Mama } \\
\text { izquierda } \\
\text { elevada }\end{array}$} \\
\hline & Ant $^{*}$ & Des $^{*}$ & Ant & Des & Ant & Des & Ant & Des & Ant & Des & Ant & Des & Ant & Des & Ant & Des \\
\hline Trombón 1 & $4^{\circ}$ & $3^{\circ}$ & - & - & $3^{\circ}$ & $1^{\circ}$ & - & - & $1^{\circ}$ & $0^{\circ}$ & $3^{\circ}$ & $0^{\circ}$ & $2^{\circ}$ & $1^{\circ}$ & $4^{\circ}$ & $2^{\circ}$ \\
\hline Trombón 2 & $4^{\circ}$ & $2^{\circ}$ & - & - & $6^{\circ}$ & $2^{\circ}$ & - & - & $2^{\circ}$ & $0^{\circ}$ & $1^{\circ}$ & $-1^{\circ}$ & $2^{\circ}$ & $1^{\circ}$ & $2^{\circ}$ & $0^{\circ}$ \\
\hline Trombón 3 & $3^{\circ}$ & $0^{\circ}$ & - & - & $3^{\circ}$ & $5^{\circ}$ & & & $1^{\circ}$ & $0^{\circ}$ & $2^{\circ}$ & $2^{\circ}$ & $3^{\circ}$ & $1^{\circ}$ & $3^{\circ}$ & $1^{\circ}$ \\
\hline Trombón 4 & $1^{\circ}$ & $1^{\circ}$ & - & - & - & - & $0^{\circ}$ & $1^{\circ}$ & $1^{\circ}$ & $0^{\circ}$ & $1^{\circ}$ & $0^{\circ}$ & $1^{\circ}$ & $0^{\circ}$ & $0^{\circ}$ & $0^{\circ}$ \\
\hline Trombón 5 & $1^{\circ}$ & $0^{\circ}$ & - & - & $4^{\circ}$ & $1^{\circ}$ & - & - & $3^{\circ}$ & $1^{\circ}$ & $2^{\circ}$ & $0^{\circ}$ & $3^{\circ}$ & $0^{\circ}$ & $2^{\circ}$ & $0^{\circ}$ \\
\hline Trombón 6 & $2^{\circ}$ & $0^{\circ}$ & - & - & $5^{\circ}$ & $2^{\circ}$ & - & - & $2^{\circ}$ & $0^{\circ}$ & $2^{\circ}$ & $0^{\circ}$ & $3^{\circ}$ & $0^{\circ}$ & $2^{\circ}$ & $0^{\circ}$ \\
\hline Trombón 7 & $2^{\circ}$ & $1^{\circ}$ & - & - & $4^{\circ}$ & $2^{\circ}$ & - & - & $0^{\circ}$ & $0^{\circ}$ & $3^{\circ}$ & $0^{\circ}$ & $3^{\circ}$ & $1^{\circ}$ & $2^{\circ}$ & $0^{\circ}$ \\
\hline Tuba & - & - & $2^{\circ}$ & $1^{\circ}$ & $1^{\circ}$ & $4^{\circ}$ & - & - & $2^{\circ}$ & $1^{\circ}$ & $2^{\circ}$ & $1^{\circ}$ & $2^{\circ}$ & $1^{\circ}$ & $2^{\circ}$ & $1^{\circ}$ \\
\hline Eufónio & 1 & $3^{\circ}$ & - & - & $1^{\circ}$ & $2^{\circ}$ & - & - & $0^{\circ}$ & $0^{\circ}$ & 4 & $2^{\circ}$ & $3^{\circ}$ & $2^{\circ}$ & $0^{\circ}$ & $0^{\circ}$ \\
\hline Ítems & $4^{\circ}$ & $3^{\circ}$ & - & - & $3^{\circ}$ & $1^{\circ}$ & - & - & $1^{\circ}$ & $0^{\circ}$ & $3^{\circ}$ & $0^{\circ}$ & $2^{\circ}$ & $1^{\circ}$ & $4^{\circ}$ & $2^{\circ}$ \\
\hline
\end{tabular}

Los resultados del estudio se pudieron ver influenciados negativamente debido al periodo vacacional a mitad de año, que llevo a detener las sesiones del programa a pesar de haber entregado planes para realizar en casa, lo cual pudo desmejorar algunos aspectos ganados inicialmente, situación que no se 
corroboró dado que, en la metodología planteada solo se propuso una valoración inicial y una final. Los resultados no pueden ser extrapolados a otras poblaciones dado el numero de la muestra.

\section{DISCUSIÓN}

Diversos estudios han demostrado la presencia de trastornos y dolencias musculo-esqueléticas en músicos. Dichos procesos investigativos surgen al asociar los mecanismos de movimiento de los intérpretes y el tiempo prolongado de interpretación que demanda el aprendizaje, la práctica y el desempeño profesional. La literatura menciona que la prevalencia de los trastornos músculo-esqueléticos en los músicos, va aproximadamente del $32 \%$ al $87 \%$, tal amplitud se da por el tipo de instrumento interpretado (11), en nuestro estudio solo $3 / 9$ sujetos presentaron dolor en zona de cintura escapular.

También se ha descrito que de todas las dolencias la mayoría se manifiestan en la edad adulta, y por alteraciones en la mecánica postural, similar a nuestro estudio, pues el $100 \%$ presentó en el momento de la evaluación inicial alteraciones en la postura, principalmente del cuadrante superior, encontrando las principales manifestaciones en la columna dorsal, cintura escapular y alineación de la cabeza.

Abreu-Ramos y colaboradores (8), identificaron como factores de riesgo, los años de interpretación e instrumento interpretado en la aparición de problemas médicos relacionados con su actividad musical, siendo el caso particular en nuestro estudio de tres instrumentistas, con más de 10 años de trabajo, presentando deficiencias posturales más evidentes.

Betancor (12) en su trabajo doctoral, determinó unas características intrínsecas, como edad, género, talla, etc., que pudieran tener influencia en la 
adquisición de desórdenes musculo-esqueléticos; dado el tamaño de la población nuestro estudio no permitió realizar correlaciones estadísticas.

El estudio de Betancor (12), también encontró que el $15,5 \%$ de los músicos de orquestas profesionales declaran ser fumadores, los porcentajes más bajos de fumadores se encuentran en instrumentistas de viento metal y son congruentes con los hallazgos encontrados, pues sólo uno de ellos refiere ser fumador. En cuanto a alimentación el mismo estudio, relacionó el bajo consumo de cigarrillo con una buena alimentación, pues la mayoría de personas que manifestaron no consumir cigarrillo consideraban tener una dieta sana y equilibrada. Dicho aspecto no coincidió con los datos que arrojó nuestro estudio pues 5 de los 9 sujetos investigados, manifestó que consume comidas rápidas; probablemente dado al hecho de que la mayoría son estudiantes procedentes de otras zonas del país y viven solos en la ciudad.

El estudio de Brandfonbrener (13) documenta los factores de riesgo más relevantes para la adquisición de alteraciones osteomusculares, para profesionales de la música. En nuestro estudio la evaluación inicial evidenció que la mayor carga musculo-esqueletica se da en el cuadrante superior, y las alteraciones resultan en estructuras como la columna cervical, dorsal, cintura escapular y extremidades superiores, entre tanto la literatura reporta la incidencia de lesiones en los profesionales de la música por zonas corporales, mencionando como principales regiones la cervical en un $27,5 \%$, de hombro en un $17 \%$ y de columna dorsal y lumbar en un $12,5 \%$, que son el resultado de la exposición constante de los segmentos mencionados (13).

En cuanto a los programas de intervención existe poca evidencia en instrumentistas de viento metal. Un estudio desarrollado por la Escuela de Fisioterapia de la Universidad de Valladolid (14), buscó evidencia de los efectos del ejercicio sobre la musculatura del tronco para la prevención el dolor en músicos de cuerdas altas, concluyendo que el trabajo en la musculatura de 
tronco no solo previene el dolor, sino mejora la condición postural convirtiéndose en una buena herramienta de prevención. En el caso de mejorías posturales con nuestro estudio se fortalecen estos planteamientos, pero se requiere una intervención con mayor población, para aceptar dicha hipótesis.

\section{CONCLUSIONES}

Con este estudio se hace evidente la necesidad de implementar estrategias de prevención en poblaciones de músicos, puesto que este programa de acondicionamiento físico influyó clínicamente en una mejor alineación postural.

Estas acciones de prevención coadyuvarían a disminuir el riesgo de aparición de dolor músculo-esquelético, además de influir positivamente en la calidad de vida, condiciones académicas y laborales de los intérpretes.

Respecto a la valoración muscular se recomienda para nuevos estudios la evaluación de músculos como el latísimo del dorso, el trapecio y los abdominales, serrato anterior, fibras medias del trapecio y pectorales. La intención al evaluar a los sinergistas es comprobar la integridad de los coadyuvantes del movimiento y que la acción no recaiga en mayor proporción sobre el músculo efector, viéndose afectada la estabilidad completa de la articulación escápulo humeral.

Finalmente podemos decir que la implementación del programa en esta población generó resultados positivos, representado en una disminución de los grados de alteración postural con respecto a los iniciales, los participantes adquirieron conocimientos en higiene y control postural y adoptaron el programa como estrategia saludable en su grupo de estudio. 


\section{REFERENCIAS}

1. Mesure, S. Postura, equilibrio y locomoción: bases neurofisiológicas. En: Viel E, coord. La marcha humana, la carrera y el salto. Barcelona: Masson; 2002. p. 75-99. Ref Type: Serial (Book,Monograph)

2. Posture Committee of the Americam Academy of Orthopaedic Surgeons. Posture and its Relationship to Orthopedic disabilities. A report of the Posture Commitee of the American Academy of Orthopaedic Surgeons. Evanstons III. American Academy of Orthopedic Surgeons:1947:1

3. Kendall's, FP; Kendall, E; Geise, P. Músculos: pruebas, funciones y dolor postural, 4 ed. Madrid: Marbán; 2000. pp. 70-118.

4. Lamberti, D. Que significa el trabajo de ensamble? Disponible en: http://www.daniellamberti.com/images/ensamble.pdf Consultado el 19 de mayo de 2016.

5. Klein-Vogelbach, S; Lahme, A; Spirgi-Gantert, I. Interpretación musical y postura corporal: Un desafio para músicos, profesores, terapeutas y médicos. Ediciones Akal, S.A. 2010 ; ISBN: 978-84-460-2746-1

6. Viaño, SM. Estudio de la relación entre las apariciones de lesiones musculo-esqueléticas en músicos instrumentistas y hábitos de actividad física $y$ vida diaria. Disponible en: http://cienciadeporte.eweb.unex.es/congreso/04\%20val/pdf/c153.pdf Consultado el 19 de mayo de 2016.

7. Laguna, MJ. La organización del trabajo y la estructura de la empresa, elementos clave de los riesgos laborales en las orquestas sinfónicas. Disponible en: http://www.fim-musicians.org/wpcontent/uploads/libro_maria_jose_laguna1.pdf Consultado el 14 de Septiembre de 2015.

8. Abreu-Ramos, AM; Micheo, WF. Lifetime prevalence of upper-body musculoskeletal problems in a professional-level symphony orchesta: Age, gender, and instrument-specific results. Science \& Medicine, 2007; 22(3): 97 
9. Chesky, K; Devroop, KY; Ford, J. Medical problems of brass instrumentalist: prevalence rates for trumpet, trombone, French horn, and low brass. Medical Problems of performing artist, 2002; 17(2):93-98

10. Epele, J; Martinez, IC. Movimiento corporal expresivo en la ejecución solista del piano, trayectoria del la mano sobre el eje vertical: un studio de caso. Actas de ECCoM, "La experiencia musical: Cuerpo, tiempo y sonido en el scenario de nuestra Mente, 2015; Vo 2 (1):89:98. Disponible en: http://www.saccom.org.ar/actas_eccom/vol21_contenido/EPELE_MARTINEZ_12ECCoM.pdf Consultada el 17 de Mayo de 2016.

11. Paarup, HM; Baelum, J; Holm, JW; Manniche, C; Wedderkopp, N. prevalence and consequences of musculoskeletal symptoms in symphony orchestra musicians vary by gender: a croos-sectional study. BMC Musculoskelet Disord, 20011; 12:223.

12. Betancor, I. Habitos de actividad fisica en musicos de orquestas sinfónicas profesionales: Un análisis empirico de ámbito internacional. Tesis Doctoral Universidad de las Palmas de Gran Canarias, 2011.

13. Brandfonbrener, Ag. Musculoskeletal problems of instrumental musicians. Hand Clinics, 2003; 19 (2):231-239.

14. Rodriguez, S. Efectos del ejercicio sobre la musculature del tronco para la prevención del dolor en musicos de cuerdas altas. Universidad de Valladolid. Escuela Universitaria de Fisioterapia. Trabajo de grado, 2015; disponible en: http://uvadoc.uva.es/handle/10324/14243 Consultado el 10 de mayo de 2016.

Referencias totales citadas: 14

Referencias citadas correspondientes a la Rev lb CC Act Fis Dep: 2 